\title{
Concerns about SARS-CoV-2 evolution should not hold back efforts to expand vaccination
}

\section{Sarah Cobey (D), Daniel B. Larremore, Yonatan H. Grad and Marc Lipsitch (D)}

Abstract | When vaccines are in limited supply, expanding the number of people who receive some vaccine, such as by halving doses or increasing the interval between doses, can reduce disease and mortality compared with concentrating available vaccine doses in a subset of the population. A corollary of such dose-sparing strategies is that the vaccinated individuals may have less protective immunity. Concerns have been raised that expanding the fraction of the population with partial immunity to SARS-CoV-2 could increase selection for vaccine-escape variants, ultimately undermining vaccine effectiveness. We argue that, although this is possible, preliminary evidence instead suggests such strategies should slow the rate of viral escape from vaccine or naturally induced immunity. As long as vaccination provides some protection against escape variants, the corresponding reduction in prevalence and incidence should reduce the rate at which new variants are generated and the speed of adaptation. Because there is little evidence of efficient immune selection of SARS-CoV-2 during typical infections, these population-level effects are likely to dominate vaccine-induced evolution.

In an effort to reduce the number of COVID-19 cases, hospitalizations and deaths as fast as possible, the UK and Canada have adopted a policy that prioritizes administering first doses of SARS-CoV-2 vaccines widely over giving second doses to those who have received one dose, and some in the USA have discussed similar policies, including the vaccination of twice the number of individuals with two half-doses. These strategies are collectively known as 'dose-sparing' strategies, intended to maximize the proportion of the population reached quickly with some vaccine. Although much of the discussion of these strategies has been in high-income countries, it is an even more pressing question globally, where there remains an extreme vaccine shortage. Fewer than two billion doses are projected to be available by the end of 2021 through COVAX, which would cover about a quarter of the 6.4 billion residents of the countries targeted by COVAX, assuming two doses are needed ${ }^{1}$. There has been controversy about the scientific basis for dose-sparing strategies and whether they will result in better outcomes for the pandemic.

Opponents of dose-sparing strategies have raised concerns about the feasibility and legal status of dose-sparing efforts, noting that providing first doses without a short-term guarantee of a second dose could lead some individuals not to come back for a second dose, or to shortages if later vaccine supplies are delayed. Multiple modelling studies have suggested that dose-sparing strategies would reduce the burden of disease from COVID-19 $\left(\mathrm{REFS}^{2-4}\right)$. To a first approximation ${ }^{5,6}$, if individuals who receive half as much vaccine (one versus two doses, or half the quantity of antigen per dose) achieve more than half the protection from clinical infection of those given a full regimen, then spreading the vaccine among more individuals will produce greater reductions in the number of clinical infections. These reductions will be even greater if the dose-sparing regimen is at least half as good as the full regimen in reducing transmission. The above-cited references ${ }^{2-4}$ make a similar point, with additional nuance specific to the present situation.
Whereas the legal, logistical and direct epidemiological impacts of dose-sparing strategies have received robust discussion and in the above-cited cases have undergone quantitative analysis, there has been another objection that is more speculative but, if correct, perhaps more important: that dose sparing will cause a more rapid emergence and spread of vaccine-resistant genetic variants $^{7-9}$. Reports of lower vaccine efficacy against the B.1.351 variant in South Africa ${ }^{10}$ add urgency to this concern. In our view, there is an argument at least as strong to suggest that dose sparing could reduce the spread of vaccine-escape variants, rather than increase it. To be clear, we consider this to be a speculative issue on which no conclusive prediction can be made. Nevertheless, given the importance of making decisions about dose sparing with imperfect information, here we discuss how evolutionary considerations argue for, rather than militate against, dose-sparing strategies.

\section{Dose sparing could reduce disease burden}

Authorized mRNA vaccines against COVID-19 likely meet the criterion that a single dose gives at least half the protection of two doses. Direct evidence of the durability of protective immune responses months after a single dose is not yet available, but the data that do exist on the immune response after a single dose of mRNA vaccine are promising. The phase III trials of the Moderna (mRNA1273) and Pfizer-BioNTech (BNT162b2) mRNA vaccines demonstrated high vaccine efficacy in the brief period starting approximately 2 weeks after the first dose to just before the administration of the second dose $\mathrm{e}^{11,12}$. Estimates of primary dose efficacy exclude the 2 weeks immediately after the first vaccination, as it takes approximately this long to develop a de novo antibody response to a new antigen. Moderna estimated the efficacy of the vaccine in this window starting 2 weeks after the first dose to be 92.1\% (95\% CI 68.8-99.1\%) $)^{13}$, and the analogous estimate for the Pfizer-BioNTech vaccine is $92.6 \%(95 \% \text { CI } 69.0-98.3 \%)^{14}$. An observational study of early vaccination roll-out in Israel estimates that one dose of the Pfizer-BioNTech vaccine reduces symptomatic infections and all documented 
a

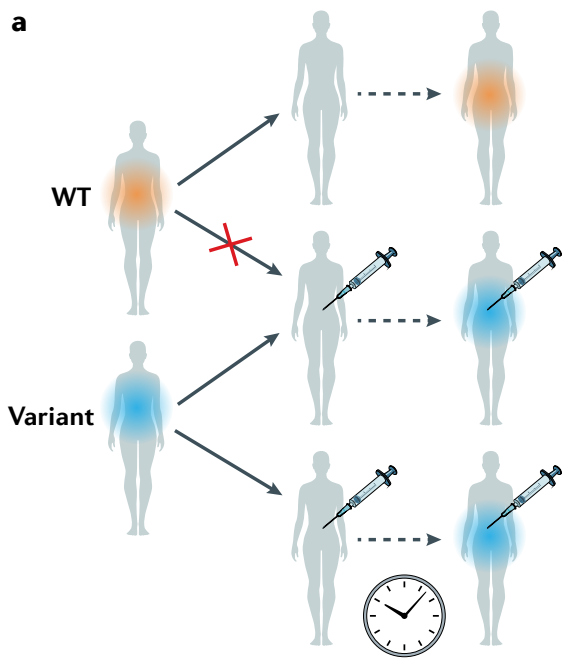

Fig. 1 | Potential vaccine-induced evolution. a | Vaccination increases the transmission advantage of an escape variant compared with wild type (WT). Here, vaccine escape is complete, allowing the variant to replace the wild type in vaccinated hosts. $\mathbf{b}$ | If residual immune protection from vaccination slows the transmission of the variant, the variant cannot spread as readily in the vaccinated population, reducing prevalence and incidence. c | Within hosts, 'intermediate' immune pressure could in
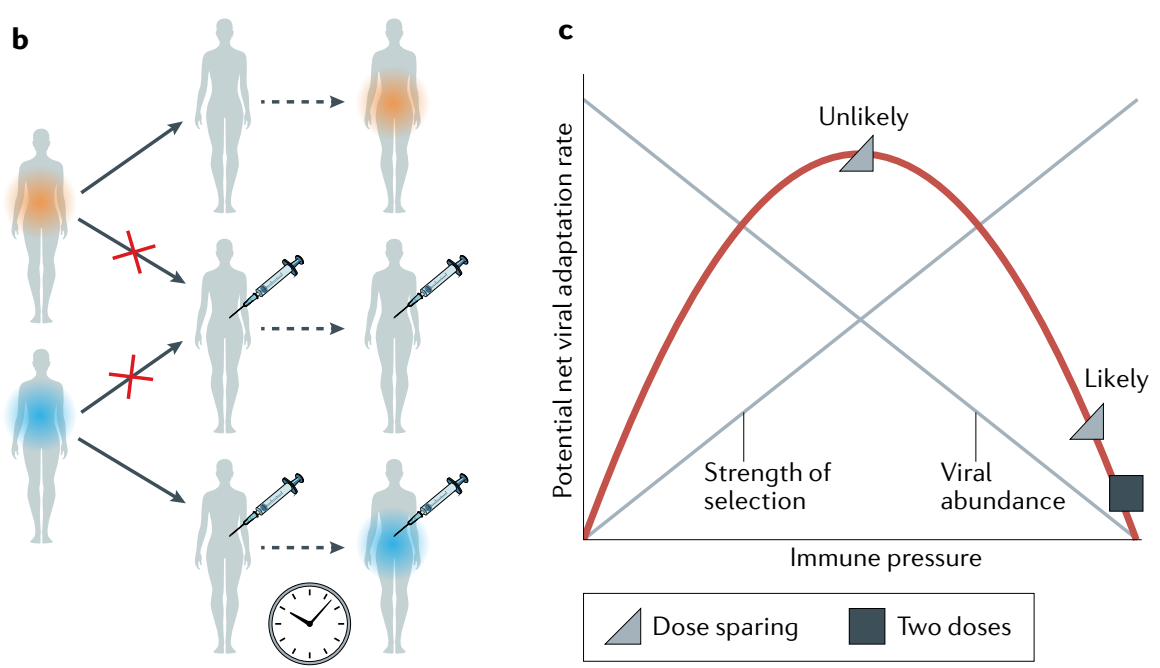

theory maximize the rate of adaptation. After two doses of vaccine, strong immune responses will likely inhibit viral replication and the emergence of escape mutations. Some have proposed that with just one dose, the rate of within-host adaptation could be high (triangle at top of the curve). We suggest that selection during COVID-19 infections is inefficient (triangle to lower right of curve). Adapted with permission from REFS 9,48 , AAAS. infections, respectively, by $57 \%(95 \% \mathrm{CI}$ $50-63 \%)$ and $46 \%$ (95\% CI 40-51\%) ${ }^{15}$.

A reduction in disease incidence and severity in vaccine recipients is the first potential benefit of dose-sparing strategies. COVID-19 vaccines can attenuate disease severity when they do not stop infection $^{11,12,16}$, a pattern sometimes observed in seasonal influenza vaccines ${ }^{17-20}$. As long as a single dose has more than half the effectiveness against disease compared with two doses, or two half doses compared with two full doses, the higher population coverage via dose-sparing regimens should protect more people against clinical infection, hospitalization and death ${ }^{2-4}$.

Another potential advantage of dose-sparing regimens is the indirect protection of others, if the vaccine decreases transmission. Reduced rates of transmission would lower the incidence of infection and prevalence. Notably, the end point in most clinical trials is symptomatic, PCR-confirmed COVID-19, and only limited data are available on the effects of vaccines on infection incidence and viral replication. Early reports from the roll-out of the Pfizer vaccine in Israel show lower nasopharyngeal viral loads in vaccine recipients who become infected ${ }^{21}$. Reductions in nasopharyngeal viral load are also observed in patients given systemically administered monoclonal antibodies ${ }^{22}$ or antibody cocktails ${ }^{23}$. Among the individuals in the randomized controlled trial of the Moderna vaccine who were swabbed before their second dose, the first dose reduced nasopharyngeal PCR positivity by $61.5 \%{ }^{12}$, and it is reasonable to think that the reduction in infectiousness may be even greater given that vaccination might cause lower viral loads among those who test positive. This is by definition more than half of the maximum reduction in infection and the attendant opportunity for transmission that one could hope to see. If this (or a greater) level of protection is sustained for many weeks after a first dose, then dose-sparing strategies would certainly reduce the prevalence of infection more than vaccination with a two-dose regimen would.

It is possible that protection against symptomatic or both symptomatic and asymptomatic infection could wane substantially in the weeks after the first dose. Although the levels of specific antibody typically fall from their peak several weeks after infection and vaccination, a study in animals suggests no reason to doubt the longevity of immune memory induced after a single dose of mRNA vaccine ${ }^{24}$. It is also possible, hypothetically, that the booster effect of a second dose of vaccine could be lower if given more than 3-4 weeks after the first dose. Again, there are few data from mRNA vaccines, but data from the chimpanzee adenovirus-vectored vaccine ChAdOx1 nCoV-19 (Oxford-AstraZeneca) indicate that, to the contrary, a longer dosing interval is associated with greater efficacy and better post-dose- 2 antibody titres ${ }^{25}$.
One might ask whether these reductions in disease incidence, severity and transmission from a dose-sparing strategy would persist in the face of variants that are less affected by the natural and/or vaccine-induced immune response. We argue that they should, because the evolution of complete escape from vaccine-induced immunity is exceedingly unlikely ${ }^{26,27}$. Thus, vaccines that reduce disease incidence or severity caused by the original (wild-type) virus are still likely to reduce disease incidence or severity caused by the escape variant. Similar effects would hold for transmission: escape variants would transmit better than the wild-type virus in a vaccinated population, but they would not transmit as readily in a vaccinated population as the wild-type virus in an unvaccinated population (FIG. 1a,b). Vaccine-induced immunity includes antibody responses, which target multiple conformational epitopes on the spike protein, and T cell responses, which target a different set of linear epitopes. Mutations that attenuate the binding of some antibodies or T cell receptors will reduce but not eliminate the level of protective immunity in the individual ${ }^{28-30}$. Consequently, it is extremely unlikely that the epidemic dynamics of a vaccine-escape variant would be identical to the dynamics of the strains against which the vaccines were tested (largely before the emergence of variants of concern). Instead, the vaccine-escape variants in vaccinated 
populations are likely to be transmitted more slowly than their ancestors were in unvaccinated populations, resulting in lower prevalence and incidence.

Taken together, these considerations argue that the protection against disease should be greater, and the reduction in prevalence of infection greater, in a dose-sparing approach whereby more individuals receive one dose than in a comparator scenario where half as many individuals receive two doses.

\section{Evolutionary considerations}

Evolution by natural selection proceeds most quickly when it has more raw material, meaning more genetic variation to work with $^{31}$, and when it is stronger, meaning in this case that the immune responses selectively promote the replication within hosts or the transmission of escape variants. Immunity can select for vaccine-escape variants of a transmissible pathogen in two ways, during infection and during transmission. Both types of selection determine the abundance of the pathogen in the population. Thus, evolutionary arguments about vaccination must consider both the propensity of immune-escape variants to spread between hosts and the rate at which these variants are generated.

\section{Reduced prevalence and transmission reduce} opportunities for emergence of resistance. The arguments above suggest that, thanks to at least some effect on transmission from one dose of vaccine, widespread use of a single dose of a mRNA vaccine will likely reduce infection prevalence compared with using the same number of doses to vaccinate half as many people, twice, at the recommended interval.

The reduced transmission and lower prevalence have several effects that individually and together tend to reduce the probability that variants with a fitness advantage, such as immune escape, will arise and spread ${ }^{32}$. The first is that with fewer infected hosts, there are fewer opportunities for new mutations to arise - reducing the available genetic variation on which selection can act. Although mutations in SARS-CoV-2 that reduce antibody binding were documented before vaccine roll-out and are thus relatively common, adaptive evolution is facilitated by the appearance of mutations and genetic rearrangements that increase the fitness benefit of other mutations $^{33-35}$. The global population size of SARS-CoV-2 is enormous, but the space of possible mutations is larger, and lowering prevalence of the virus helps to constrain the exploration of the mutational space. Other benefits arise in cases where a small fraction of hosts drives most transmission, as is the case for SARS-CoV-2 (REFS $\left.{ }^{36,37}\right)$ and where the effective reproductive number is low. Selection operates less effectively under these conditions: beneficial mutations will more often be lost by chance, and variants with beneficial mutations are less certain to rise to high frequencies in the population $^{38-42}$. More research is clearly needed to understand the precise impact of vaccination on SARS-CoV-2 evolution, but multiple lines of evidence suggest that vaccination strategies that reduce viral prevalence would reduce rather than accelerate the rate of adaptation, including antigenic evolution and, thus, the incidence of successful mutations over the long term.

In evaluating the potential impact of expanded vaccine coverage facilitated by dose-sparing regimens on the transmission of escape variants, it is necessary to compare the alternative scenario, in which fewer individuals are vaccinated (but a larger proportion receive two doses) and more people recover from natural infection. The immunity that develops during the course of natural infection and the immune response that inhibits repeat infection also impose selection pressure on the virus. Although natural infection evokes immune responses to a broader set of antibody and $\mathrm{T}$ cell targets than vaccination, antibodies to the spike protein are likely a major component of protection after either kind of exposure ${ }^{43-45}$, and genetic variants that escape polyclonal sera after natural infection have already been identified ${ }^{46,47}$. Studies comparing the effectiveness of past infection and vaccination on protection and transmission are ongoing. If protective immunity, and specifically protection against transmission, from natural infection is weaker than that from one dose of vaccination, the rate of spread of escape variants in populations with infection-induced immunity could be higher than in those with vaccine-induced immunity. In this case, an additional advantage of increasing vaccine coverage through dose-sparing regimens might be a reduction in the selective pressure from infection-induced immunity.

\section{Within hosts, dose-sparing regimens are unlikely to promote immune escape. As} has long been noted ${ }^{48}$, immune responses reduce viral growth, which reduces genetic variation, creating a 'Goldilocks' situation for adaptation: too little immune response means not much selective pressure to escape immunity, and too much immune response shuts down viral replication before escape variants can be generated. In theory, at intermediate levels of immunity, there is enough viral replication to generate escape variants and enough selection pressure to amplify those variants so that they grow to high frequency and may be transmitted to others (FIC. 1c).

In the simplest terms, the concern that dose-sparing strategies will enhance the spread of immune-escape mutants postulates that individuals who receive a single dose of vaccine are those with the intermediate, 'just right' level of immunity, more likely to evolve escape variants than those with zero or two doses ${ }^{7,9}$. Hypothetically, this intermediate level of immunity could arise weeks to months after vaccination, after initial immune responses have waned, and would have been avoided had the second dose been received earlier. Similarly, for the half-dose strategy, the postulate is that an individual with two half-doses has immunity closer to just right levels than an individual with no doses or two full doses.

There is no particular reason to believe this is the case. Strong immune responses arising from past infection or vaccination will clearly inhibit viral replication, preventing infection and thus within-host adaptation. But it is unclear whether weaker immune responses that do permit viral replication should impose much selective pressure. Unlike the situation in chronic infections such as HIV, relatively few generations of replication and thus selection occur in hosts experiencing acute infections such as COVID-19. Most transmission occurs within a day or two of peak viral load, near the onset of symptoms $s^{49,50}$. The small founding populations and short time to peak load afford little time for escape variants to appear via mutation and rise to appreciable abundance, especially if viral loads are suppressed owing to residual immunity from vaccination ${ }^{51-56}$. Past work on influenza has found no evidence of selection for escape variants during infection in vaccinated hosts $^{57}$. Instead, evidence suggests that it is immunocompromised hosts with prolonged influenza infections and high viral loads whose viral populations show high diversity and potentially adaptation ${ }^{58,59}$, a phenomenon also seen with SARS-CoV-2 $\left(\mathrm{REFS}^{60-63}\right)$. It seems likely, given its impact on disease, that vaccination could shorten such infections, and there is limited evidence already that vaccination reduces the amount of virus present in those who do become infected after vaccination ${ }^{21}$.

The implication is that because within-host selection tends to be inefficient, 
the emergence by mutation and onward transmission of vaccine-escape variants is not necessarily more likely in vaccinated hosts than in unvaccinated ones, including individuals with immunity from natural infection. Instead, the strongest selection for vaccine-escape mutants occurs via transmission.

\section{Discussion}

We argue above that dose-sparing regimens will not necessarily increase the risk of vaccine escape and might even lower it. Moreover, even under worst-case evolutionary scenarios, residual immunity from dose-sparing strategies should reduce the burden of COVID-19 disease. We propose that this residual immunity would in general not be expected to promote the evolution of escape variants because selection of de novo mutations is inefficient during individual infections, and residual immunity from dose-sparing strategies should slow transmission of SARS-CoV-2 overall. This, in turn, will slow the rate of adaptation and the possibilities for further escape. This evolutionary logic implies that any measures to reduce the rate of transmission, not only through dose-sparing regimens, could reduce the rate of escape from vaccine or naturally induced immunity and the emergence of more transmissible variants. Our argument therefore stands in contrast to previous concerns about selection for escape variants, which have largely ignored population-level dynamics, while positing strong selection within hosts $^{7,9}$.

Although they are based on the best available evidence, these conclusions are necessarily tentative. They rely on the notable assumptions that partial or delayed dosing can be at least half as effective as full dosing and that vaccines will continue to offer some protection against the transmission of escape variants. There is an urgent need for molecular epidemiological studies and quantitative modelling to better understand the dynamics of immunity after infection and vaccination, including how immunity relates to protection against disease and transmission. Longitudinal studies that track natural infection among vaccinated and unvaccinated individuals are useful to evaluate the strength and durability of protection against disease and subclinical infections. By measuring shedding duration and intensity, such studies can also indirectly estimate the impact of immunity on transmission, although transmission is better studied in household studies and cluster-randomized trials. A full understanding of the epidemiological and evolutionary impacts of vaccination requires reconciling individual observations with population patterns. Vaccine effectiveness against specific viral lineages can be measured by outpatient surveillance of clinical infections in vaccinated and unvaccinated individuals, as occurs for seasonal influenza ${ }^{64-66}$. Expanded genomic surveillance would also enable comparison of lineage dynamics and disease rates in areas with different vaccination coverage ${ }^{67}$. Combined, these measures could drive quantitative assessments of vaccination strategies and evaluate the truth of our assumptions.

An important caveat to our argument is that we assume that mutations that confer vaccine escape are not exclusively linked to other fitness-enhancing mutations. In other words, we assume that phenotypic traits are independent. For instance, if a mutation that confers a doubling in transmission rate, independently of immune recognition, arose in a vaccine-escape variant, and if it only arose on this genetic background, then vaccination would accelerate the speed with which this variant displaced resident strains. Hitchhiking mutations that confer resistance to antivirals in influenza have spread unexpectedly through selection for faster replication ${ }^{68}$ or a putative immune-escape variant. These situations are challenging to predict. But, although SARS-CoV-2 vaccine-escape variants such as B.1.351 are associated with mutations that might increase transmissibility, such as N501Y, other lineages, such as B.1.1.7, also show high rates of transmission without comparable advantages against vaccines. Thus, we suspect vaccination will not accelerate the evolution of more transmissible variants - they are spreading regardless - and there are theoretical reasons to expect that vaccination will prevent their continued emergence. We note that the first putative vaccine-escape variant, B.1.351, and the possible immune-escape variant P1 spread in the presence of little or no vaccine-induced immunity.

In practice, it may be wise for particular subpopulations to maintain a typical vaccination schedule even when dose sparing is used in the broader population. For instance, in balancing risks, older adults or other highly vulnerable groups might maintain a two-dose regimen with phase III trial dose spacing, given their increased risk of severe disease should they become infected. It could also be useful to maintain a more conservative regimen in immunocompromised individuals, given their implication in the generation of variants $\mathrm{s}^{60-63}$.

The pandemic forces difficult choices under scientific uncertainty. There is a risk that appeals to improve the scientific basis of decision-making will inadvertently equate the absence of precise information about a particular scenario with complete ignorance, and thereby dismiss decades of accumulated and relevant scientific knowledge. Concerns about vaccine-induced evolution are often associated with worry about departing from the precise dosing intervals used in clinical trials. Although other intervals were investigated in earlier immunogenicity studies, for mRNA vaccines, these intervals were partly chosen for speed and have not been completely optimized. They are not the only information on immune responses. Indeed, arguments that vaccine efficacy below 95\% would be unacceptable under dose-sparing regimens of mRNA vaccines imply that campaigns with the other vaccines that are estimated to have a lower efficacy pose similar problems. Yet few would advocate that these vaccines should be withheld in the thick of a pandemic, or roll-outs slowed to increase the number of doses that can be given to a smaller group of people. We urge careful consideration of scientific evidence to minimize lives lost.

Finally, the global nature of the SARS-CoV-2 pandemic and continuing movement of variants across continents limits both the disadvantages cited by opponents of dose sparing and the advantages highlighted here. It may be short-sighted to imagine that the policy of any one country can have a large influence on the global evolution of the virus.

\section{Conclusion}

We propose that dose-sparing strategies, which could have large public health benefits, not be dismissed out of concern that they might promote immune escape in SARS-CoV-2. In fact, multiple lines of evidence suggest that expanded vaccination coverage could reduce the rate of immune escape, providing an additional benefit of dose sparing beyond its immediate impact on disease. These beneficial effects hinge on the assumption that vaccination provides some protection against variants of SARS-CoV-2, or, in other words, that vaccine effectiveness against the variants is not zero under dose-sparing regimens. Another requirement is that other fitness-enhancing mutations, such as those allowing for faster replication, do not exclusively occur in genomes with 
vaccine-escape mutations. Both of these assumptions appear to be met at present. We encourage research to refine our understanding of vaccine effectiveness, immune pressure and the evolutionary dynamics of SARS-CoV-2, and to investigate this problem more thoroughly.

Sarah Cobey $\mathbb{D D}^{\prime \otimes}$, Daniel B. Larremore $e^{2,3}$,
Yonatan H. Grad ${ }^{4}$ and Marc Lipsitch $\mathrm{ID}^{4,5}$

'Department of Ecology and Evolution, University of Chicago, Chicago, IL, USA.

2 Department of Computer Science, University of Colorado Boulder, Boulder, CO, USA.

${ }^{3}$ BioFrontiers Institute, University of Colorado Boulder, Boulder, CO, USA

'Department of Immunology and Infectious Diseases, Harvard T. H. Chan School of Public Health, Boston, MA, USA.

${ }^{5}$ Center for Communicable Disease Dynamics, Department of Epidemiology, Harvard T. H. Chan School of Public Health, Boston, MA, USA.

凶e-mail: cobey@uchicago.edu

https://doi.org/10.1038/s41577-021-00544-9

Published online 1 April 2021

1. World Health Organization. COVAX Global Supply Forecast (WHO, 2021)

2. Tuite, A. R., Zhu, L., Fisman, D. N. \& Salomon, J. A. Alternative dose allocation strategies to increase benefits from constrained COVID-19 vaccine supply. Ann. Intern. Med. https://doi.org/10.7326/M20-8137 (2021).

3. Barnabas, R. V. $\&$ Wald, A. A public health COVID-19 vaccination strategy to maximize the health gains for every single vaccine dose. Ann. Intern. Med. https:// doi.org/10.7326/M20-8060 (2021)

4. Paltiel, A. D. Zheng, A. \& Schwartz, J. L. Speed versus efficacy: quantifying potential tradeoffs in COVID-19 vaccine deployment. Ann. Intern. Med. https://doi.org/ 10.7326/M20-7866 (2021).

5. Riley, S., Wu, J. T. \& Leung, G. M. Optimizing the dose of pre-pandemic influenza vaccines to reduce the infection attack rate. PLoS Med. 4, e218 (2007).

6. Wu, J. T., Peak, C. M., Leung, G. M. \& Lipsitch, M. Fractional dosing of yellow fever vaccine to extend supply: a modelling study. Lancet 388, 2904-2911 (2016).

7. Bieniasz, P. The case against delaying SARS-CoV-2 mRNA vaccine boosting doses. Clin. Infect. Dis. https:/ doi.org/10.1093/cid/ciab070 (2021).

8. New and Emerging Respiratory Virus Threats Advisory Group (NERVTAG). SARS-CoV-2 immunity-escape variants. gov.uk https://www.gov.uk/government/ publications/sars-cov-2-immunity-escape-variants-7january-2021 (2021).

9. Saad-Roy, C. M. et al. Epidemiological and evolutionary considerations of SARS-CoV-2 vaccine dosing regimes. Science https://doi.org/10.1126/science.abg8663 (2021).

10. Madhi, S. A. et al. Safety and efficacy of the ChAdOx 1 nCoV-19 (AZD1222) Covid-19 vaccine against the B. 1.351 variant in South Africa. Preprint at medRxiv https://doi.org/10.1101/2021.02.10.21251247 (2021).

11. Polack, F. P. et al. Safety and efficacy of the BNT162b2 mRNA Covid-19 vaccine. N. Engl. J. Med. 383 . 2603-2615 (2020)

12. Baden, L. R. et al. Efficacy and safety of the mRNA1273 SARS-CoV-2 vaccine. N. Engl. J. Med. 384, 403-416 (2020)

13. FDA. FDABriefing Document Moderna COVID-19 Vaccine, Vaccines and Related Biological Products Advisory Committee (ModernaTX, Inc., 2020)

14. Skowronski, D. M. \& De Serres, G. (Correspondence) Safety and efficacy of the BNT162b2 mRNA Covid-19 vaccine. N. Engl. J. Med. https://doi.org/10.1056/ NEJMc2036242 (2021)

15. Dagan, N. et al. BNT $162 \mathrm{~b} 2$ mRNA Covid-19 vaccine in a nationwide mass vaccination Setting. $N$. Engl. J. Med. https://doi.org/10.1056/nejmoa2101765 (2021).
16. Voysey, M. et al. Safety and efficacy of the ChAdOx1 $n C o V-19$ vaccine (AZD 1222) against SARS-CoV-2: an interim analysis of four randomised controlled trials in Brazil, South Africa, and the UK. Lancet 397, 99-111 (2021).

17. Tenforde, M. W. et al. Influenza vaccine effectiveness against hospitalization in the United States, 2019-2020. J. Infect. Dis. https://doi.org/10.1093/ infdis/jiaa800 (2021)

18. Thompson, M. G. et al. Influenza vaccine effectiveness in preventing influenza-associated intensive care admissions and attenuating severe disease amon adults in New Zealand 2012-2015. Vaccine 36, 5916-5925 (2018).

19. Arriola, C. S. et al. Does influenza vaccination modify influenza severity? Data on older adults hospitalized with influenza during the 2012-2013 season in the United States. J. Infect. Dis. 212, 1200-1208 (2015).

20. Arriola, C. et al. Influenza vaccination modifies disease severity among community-dwelling adults hospitalized with influenza. Clin. Infect. Dis. 65 1289-1297 (2017).

21. Levine-Tiefenbrun, M. et al. Decreased SARS-CoV-2 viral load following vaccination. Preprint at medRxiv https://doi.org/10.1101/2021.02.06.21251283 (2021).

22. Chen, P. et al. SARS-CoV-2 neutralizing antibody LYCoV555 in outpatients with Covid-19. N. Engl. J. Med. 384, 229-237 (2021).

23. Weinreich, D. M. et al. REGN-COV2, a neutralizing antibody cocktail, in outpatients with Covid-19. N. Engl. J. Med. 384, 238-251 (2021).

24. Pardi, N. et al. Nucleoside-modified mRNA vaccines induce potent $\mathrm{T}$ follicular helper and germinal cente B cell responses. J. Exp. Med. 215, 1571-1588 (2018).

25. Voysey, M. et al. Single-dose administration and the influence of the timing of the booster dose on immunogenicity and efficacy of ChAdOx $1 \mathrm{nCoV}-19$ (AZD 1222) vaccine: a pooled analysis of four randomised trials. Lancet 397, 881-891 (2021).

26. Kennedy, D. A. \& Read, A. F. Why does drug resistance readily evolve but vaccine resistance does not? Proc. Biol. Sci. 284, 20162562 (2017)

27. Tarke, A. et al. Negligible impact of SARS-CoV-2 variants on $\mathrm{CD}^{+}$and $\mathrm{CD} 8^{+} \mathrm{T}$ cell reactivity in COVID-19 exposed donors and vaccinees. Preprint at bioRxiv https://doi.org/10.1101/2021.02.27.433180 (2021).

28. Greaney, A. J. et al. Complete mapping of mutations to the SARS-CoV-2 spike receptor-binding domain that escape antibody recognition. Cell Host Microbe 29 44-57.e9 (2021).

29. Wang, Z. et al. mRNA vaccine-elicited antibodies to SARS-CoV-2 and circulating variants. Nature https:/ doi.org/10.1038/s41586-021-03324-6 (2021).

30. Liu, Z. et al. Landscape analysis of escape variants identifies SARS-CoV-2 spike mutations that attenuate monoclonal and serum antibody neutralization. Preprint at bioRxiv https://doi.org/10.1101/ 2020.11.06.372037 (2020).

31. Fisher, R. A. The Genetical Theory of Natural Selection: A Complete Variorum Edition (University Press, 1999)

32. Wen, F. T., Malani, A. \& Cobey, S. The beneficial effects of vaccination on the evolution of seasonal influenza. Preprint at bioRxiv https://doi.org/10.1101/162545 (2020).

33. Gong, L. I., Suchard, M. A. \& Bloom, J. D. Stability mediated epistasis constrains the evolution of an influenza protein. eLife 2, e00631 (2013).

34. $\mathrm{Wu}, \mathrm{N}$. C. et al. Systematic identification of $\mathrm{H} 274 \mathrm{Y}$ compensatory mutations in influenza A virus neuraminidase by high-throughput screening. J. Virol. 87, 1193-1199 (2013)

35. Starr, T. N. \& Thornton, J. W. Epistasis in protein evolution. Protein Sci. 25, 1204-1218 (2016)

36. Adam, D. C. et al. Clustering and superspreading potential of SARS-CoV-2 infections in Hong Kong. Nat. Med. 26, 1714-1719 (2020)

37. Xu, X.-K. et al. Reconstruction of transmission pairs for novel coronavirus disease 2019 (COVID-19) in mainland china: estimation of superspreading events, serial interval, and hazard of infection. Clin. Infect. Dis. 71, 3163-3167 (2020)

38. Desai, M. M., Fisher, D. S. \& Murray, A. W. The speed of evolution and maintenance of variation in asexual populations. Curr. Biol. 17, 385-394 (2007).

39. Patwa, Z. \& Wahl, L. M. The fixation probability of beneficial mutations. J. R. Soc. Interface 5 1279-1289 (2008).
40. Otto, S. P. $\&$ Whitlock, M. C. The probability of fixation in populations of changing size. Genetics 146 723-733 (1997).

41. Desai, M. M. \& Fisher, D. S. Beneficial mutationselection balance and the effect of linkage on positive selection. Genetics 176, 1759-1798 (2007).

42. Kimura, M. Some problems of stochastic processes in genetics. Ann. Math. Stat. 28, 882-901 (1957).

43. Addetia, A. et al. Neutralizing antibodies correlate with protection from SARS-CoV-2 in humans during a fishery vessel outbreak with a high attack rate. J. Clin. Microbiol. 58, e02107-20 (2020).

44. Zost, S. J. et al. Potently neutralizing and protective human antibodies against SARS-CoV-2. Nature $\mathbf{5 8 4}$, 443-449 (2020).

45. Steffen, T. L. et al. The receptor binding domain of SARS-CoV-2 spike is the key target of neutralizing antibody in human polyclonal sera. Preprint at bioRxiv https://doi.org/10.1101/2020.08.21.261727 (2020).

46. Weisblum, Y. et al. Escape from neutralizing antibodies by SARS-CoV-2 spike protein variants. eLife $\mathbf{9}$, e61312 (2020).

47. Andreano, E. et al. SARS-CoV-2 escape in vitro from a highly neutralizing COVID-19 convalescent plasma. Preprint at bioRxiv https://doi.org/10.1101/ 2020.12.28.424451 (2020).

48. Grenfell, B. T. et al. Unifying the epidemiological and evolutionary dynamics of pathogens. Science 303 , 327-332 (2004).

49. He, X. et al. Temporal dynamics in viral shedding and transmissibility of COVID-19. Nat. Med. 26, 672-675 (2020).

50. Li, F. et al. Household transmission of SARS-CoV-2 and risk factors for susceptibility and infectivity in Wuhan: a retrospective observational study. Lancet Infect. Dis. https://doi.org/10.1016/S1473-3099(20)30981-6 (2021).

51. Morris, D. H. et al. Asynchrony between virus diversity and antibody selection limits influenza virus evolution. eLife 9, e62105 (2020).

52. McCrone, J. T et al. Stochastic processes constrain the within and between host evolution of influenza virus. eLife 7, e35962 (2018)

53. Valesano, A. L. et al. Temporal dynamics of SARS CoV-2 mutation accumulation within and across infected hosts. Preprint at bioRxiv https://doi.org/ 10.1101/2021.01.19.427330 (2021).

54. Martin, M. A. \& Koelle, K. Reanalysis of deepsequencing data from Austria points towards a small SARS-COV-2 transmission bottleneck on the order of one to three virions. Preprint at bioRxiv https://doi.org 10.1101/2021.02.22.432096 (2021).

55. Lythgoe, K. A. et al. SARS-CoV-2 within-host diversity and transmission. Science https://doi.org/10.1126/ science.abg0821 (2021).

56. Braun, K. M. et al. Transmission of SARS-CoV-2 in domestic cats imposes a narrow bottleneck. PLoS Pathog. 17, e1009373 (2021).

57. Debbink, K. et al. Vaccination has minimal impact on the intrahost diversity of $\mathrm{H} 3 \mathrm{~N} 2$ influenza viruses. PLoS Pathog. 13, e1006194 (2017).

58. Xue, K. S. et al. Parallel evolution of influenza across multiple spatiotemporal scales. eLife 6, e26875 (2017).

59. Xue, K. S., Moncla, L. H., Bedford, T. \& Bloom, J. D. Within-host evolution of human influenza virus. Trends Microbiol. 26, 781-793 (2018)

60. Choi, B. et al. Persistence and evolution of SARS-CoV-2 in an immunocompromised host. N. Engl. J. Med. 383, 2291-2293 (2020)

61. Kemp, S. A. et al. Neutralising antibodies in Spike mediated SARS-CoV-2 adaptation. Preprint at medRxiv https://doi.org/10.1101/2020.12.05.20241927 (2020).

62. Ko, S. H. et al. High-throughput, single-copy sequencing reveals SARS-CoV-2 spike variants coincident with mounting humoral immunity during acute COVID-19. Preprint at bioRxiv https://doi.org/ 10.1101/2021.02.21.432184 (2021).

63. Clark, S. A. et al. SARS-CoV-2 evolution in an immunocompromised host reveals shared neutralization escape mechanisms. Cell https://doi.org/10.1016/ j.cell.2021.03.027 (2021)

64. Flannery, B. et al. Spread of antigenically drifted influenza $A(H 3 N 2)$ viruses and vaccine effectiveness in the United States during the 2018-2019 season. J. Infect. Dis. 221, 8-15 (2020).

65. Skowronski, D. M. et al. A perfect storm: impact of genomic variation and serial vaccination on low influenza vaccine effectiveness during the 2014-2015 season. Clin. Infect. Dis. 63, 21-32 (2016). 
66. Skowronski, D. M. et al. Influenza vaccine effectiveness by $\mathrm{A}(\mathrm{H} 3 \mathrm{~N} 2)$ phylogenetic sub-cluster and prior vaccination history: 2016-17 and 2017-18 epidemics in Canada. J. Infect. Dis. https://doi.org/10.1093/infdis/ jiaa138 (2020).

67. Wen, F. T., Bell, S. M., Bedford, T. \& Cobey, S. Estimating vaccine-driven selection in seasonal influenza. Viruses 10, 509 (2018)

68. Bloom, J. D., Gong, L. I. \& Baltimore, D. Permissive secondary mutations enable the evolution of influenza oseltamivir resistance. Science 328, 1272-1275 (2010).

\section{Acknowledgements}

The authors thank E. T. Martin, J. Bloom, P. Pennings,

C. Illingworth and M. Mina for comments. S.C. was supported

by the NIAID Collaborative Influenza Vaccine Innovation Centers
(CIVIC) contract 75N93019C00051. D.B.L. and M.L. were supported in part by the SeroNet programme of the National Cancer Institute (1U01CA261277-01). The content is solely the responsibility of the authors and does not necessarily represent the official views of the National Institutes of Health. D.B.L. was supported in part through the MIDAS Coordination Center (MIDASNI2020-2) by a grant from the National Institute of General Medical Science (3U24GM132013-02S2). M.L. was supported by a gift from the Morris-Singer Foundation.

Author contributions

The authors contributed equally to all aspects of the article.

\section{Competing interests}

M.L. has received honoraria/consulting from Sanofi-Pasteur, Bristol Myers Squibb and Merck; receives research funding (institutional) from Pfizer; and has provided unpaid scientific advice to Pfizer, Janssen, AstraZeneca, One Day Sooner and Covaxx (United Biomedical). Y.H.G. has received consulting fees from GSK and Merck not related to the subject of this manuscript, and research funding (institutional) from Pfizer and Merck also unrelated to this work. D.B.L. and S.C. declare no competing interests.

\section{Peer review information}

Nature Reviews Immunology thanks A. Iwasaki and the other, anonymous, reviewer(s) for their contribution to the peer review of this work.

\section{Publisher's note}

Springer Nature remains neutral with regard to jurisdictional claims in published maps and institutional affiliations.

(c) Springer Nature Limited 2021 